\title{
Unrelenting challenges for freaque wave studies in ocean coastal regions: defining the phenomena
}

\author{
P. C. Liu \\ NOAA Great Lakes Environmental Research Laboratory, USA
}

\begin{abstract}
In this paper we point out the lesser known or underexplored aspects of freaque waves and what challenges we are facing. The current study of freaque waves has been an active research field over the last two decades or so. There have been significant advancements especially in connection with the study of nonlinear physics. We have explored what we do or do not know; in this paper, we present the unrelenting challenges that we are still facing.

Keywords: ocean waves, freaque waves, the phenomena, freak waves, rogue waves.
\end{abstract}

\section{Introduction}

The freaque wave has been in existence probably as long as the world's oceans have existed. But it was not until Draper [1] first calling it a "freak wave" did its existence ever being academically acknowledged. And it took still over another two decades when Draupner platform in the North Sea recorded the famed wave profile on 1995 New Year's Day (Haver [2]) that everyone immediately recognized as the shape of a freaque wave did the academic world beginning to embark on freaque wave studies.

Dias et al. [3] made an interesting observation regarding freaque waves: ". . . once part of the folklore, they now made the news each time an observation is made." Indeed we have probably heard more media reports on freaque wave cases in the first decade of the 21 st century than in all of the 20th century years combined. But a curious phenomenon crops up here: out of all the freaque wave encounters that were reported, do we really have a clear notion as to what was happening out there? Most likely, we know something unexpected occurred 
but not much else. So, in regard to freaque waves that are happening in the ocean and lakes every day, we think there is more that we do not know about them than what we do know. One of Confucius' analects says "Know what you do know, and recognize what you do not know, that then is true knowledge" may be applicable here. In this paper, we present an exploration on the aspects of the freaque waves that we do not seem to have clear answers for as our unrelenting challenges. Of course, these are personal opinions; presumably they will not be shared by all. Some may consider these issues uninteresting or even unscientific or trivial, but they are the topics that we do not know and should not remain obscured.

\section{Freaque waves}

Let's start with some nomenclature here. The term "freaque wave" is a portmanteau word that blends the two commonly used words "freak" and "rogue" in reference to "freak or rogue waves" frequently used. Actually, there are also terms like killer waves, extreme waves, monster waves, giant waves, abnormal waves, sneaker or sleeper waves being used. We wish for "freaque waves" to represent all of the above except extreme waves. Because the main feature of the phenomenon is unexpectedness, whereas every wave recording has a local maximum or extreme, extreme waves really do not explicitly belong to the "unexpectedness" category. At any rate, this usage merely represents a personal preference for convenience.

\section{What do we know?}

Three years ago, Liu et al. [4] first asked the question: "What do we know about freaque waves in the ocean and lakes and how do we know it?" There they made an objective examination of our present state of knowledge on freaque waves in the ocean and lakes from three separate perspectives:

- testimonial - from eyewitness accounts of actual encounters;

- $\quad$ empirical - from available in-situ wave measurements;

- conjectural - from academic theoretical formulations.

Thereby, they subjectively surmised that "we do not know very much about freaque waves in the ocean and lakes!" Clearly, there is really no surprise there. The three perspectives are nature settings where our basic knowledge bases are summarily developed from. However, saying we do not know very much is too broad a statement to be of substance. Here we hope that we can provide some specifics!

\section{What do we not know?}

Liu et al. [5] also asked the opposite question regarding what we do not know. To begin with, it is obvious that we have not yet been able to grasp the basic 
fundamentals of freaque waves: where, when, how, or why a freaque wave occurs in the ocean and lakes. This is of interest to the scientific community at large doing freaque waves research and also to the general public. The research community has endeavored to understand the where, when, how, or why in recent years with considerable success, we can only present our personal viewpoints that may or may not be valid, and some may also be on the contrarian side. Nevertheless, here are some of our thoughts:

\section{- Do we have a viable definition for the phenomena yet?}

What is a freaque wave? That is a question still frequently being asked without a satisfactory answer. The standard de facto definition is that a wave with its height greater than twice the significant wave height. Some call it an ad hoc definition for freaque waves that is universally accepted. But it is a conditional definition, basically risen from the availability of a wave data record recorded from a single point wave gage with the presumption the ocean surface should necessarily be Gaussian random process and the wave heights follow a Rayleigh distribution. These simple conditions for a wave height to be considered freaque have become moot, and no one really pays attention to them.

In addition to these specified conditions largely being ignored, most of the reported freaque wave occurrences in the ocean, on the other hand, usually do not have the benefit of an available wave recording to substantiate that the definition is adhered to. So in reality, a reported freaque wave encounter will be most likely just in the eyes of an eyewitness - with or without a need to check or specify the conditional definition. Thus, for the most part, the well known definition has been rendered inoperative and there is no consensus that a correct definition of a freaque wave will ever be reached. So, the state of freaque waves remains that everyone recognizes their existence and that it is happening out there, but not much anything else is known. In other words we'll know it happened when we see it, but we cannot effectively define it!

\section{- Does the well-known plot of the Draupner platform 1995 New Year's Day wave data truly represent the same kind of freaque waves widely reported to have been occurring in the ocean and lakes that caused disasters and damage?}

The discovery of the wave data recorded on the Statoil's Draupner platform in the North Sea from a laser lookdown sensor on 1995 New Year's day, now widely known as the Draupner wave, is probably the primary reason that jump started modern ocean freaque wave research and kindled excitement over the past two decades or so, especially in the nonlinear physics communities.

As the Draupner wave becomes the typical profile for a freaque wave, ocean scientists around the world all seem to be able to find similar kind of 
recordings in their data collections. It is for real; Draupner-type freaque waves happen all around the world, and excitement abounds. But amidst all the "Draupner" excitement, some questions beg for an answer. First and foremost, were all the freaque waves of the past that caused so many maritime disasters and tragedies the kind of Draupner wave? Alternatively, is a typical Draupner wave the kind of wave that was once called the "Killer Wave"?

Perhaps the more intriguing fact of the Draupner wave recorded from the Draupner Platform is that it was not immediately recognized until discovered a couple of days later (Haver [2]). It happened on the giant platform where workers present were not aware that it was happening on the early afternoon of the 1995 New Year's Day. There was a storm and part of the platform was damaged. Were the damages really caused by the Draupner wave per se? No one nearby noticed it was happening.

We are all familiar with the recording plot of the Draupner wave, and have seen similar recording plots from many other parts of the world. What we do not know for certain is whether or not this kind of wave profile is actually capable of the kind of damage and destruction that we would expect. We know the waves exist, and we recognize the disastrous results. We are not certain if there is an actual substantiation of the inference!

Ideally, this dilemma can only be resolved by actual measurement. Is it too much to expect to have some actual measurement at the onset of the freaque wave occurrence? This, of course, is not really too farfetched, we just need some well planned and better conceived measurement programs. Unless we have actual measurements to substantiate, educated speculations are always unconvincing for some and at the same time can face endless questions.

\section{- Are there different kinds of freaque waves?}

No one can really suppose that freaque wave occurrence is a unique phenomenon. There are deep ocean freaque waves, and there are nearshore freaque waves, their processes are clearly different. Nonlinear physicists, on the other hand, are concerned with only one kind - the kind that resembles the Draupner wave profile. Most of the advancements in academic freaque wave research in recent years were in the nonlinear physics world, and they all seem more or less to pertain to the appearance of Draupner waves.

Akhmediev et al. [6] have a very interesting title: "Waves that appear from nowhere and disappear without a trace." That descriptive title "appear from nowhere and disappear without a trace" seems to describe the kind of freaque wave most people visualize whenever the term freaque wave is mentioned. But that description does not seem to match the Draupner wave profile. Of course, Akhmediev et al.'s [6] solution is still that of a onedimensional Draupner wave with an envelope. An impressive spatial simulation that well-suited the descriptions "appear from nowhere and disappear without a trace" is given by Hohmann et al. [7] through their 
microwave study and their simulation in truly three-dimensional ocean space can be viewed at Youtube (http://www.youtube.com/watch?v=hUX8P7Q2KU).

Long before modern freaque wave research, when freaque waves were still considered as folklore, there were two frequently mentioned kinds that they encountered: a wall of water, and a hole in the sea. These are two distinct cases of freaque waves that are extremely dangerous if encountered in the deep ocean. We don't know how many tragedies and lives have been lost by theses two notorious kinds of freaque waves, only a few have been fortunate enough to tell the story. Since they are not to be believed, clearly no details were preserved. Now fast forward to the present, we hear about freaque wave occurrences here and there, from somewhere around the world from the news reports. A very recent theoretical effort by Chabchoub et al. [8] on the 1-D "a hole in the sea" case has presented with some water wave tank demonstration.

But, for the time being, whenever we hear of a freaque wave encounter, the things that we can be quite certain of are that we don't know what happened, and we don't know how and why it happened.

There must be different kinds of freaque waves happening out there. In the end, the only way to answer these unascertained questions regarding freaque waves is to make long-term systematic wave measurements!

\section{- How often does a freaque wave occur?}

After hearing or seeing a Draupner wave for the first time, many of us wondered how often this kind of freaque wave case generally happens. Not an unreasonable question. Unfortunately, Statoil installed the wave measurement instrument only for a short time, it was not intended as for long-term measurements, and there is no data available to realistically assess how often the kind of Draupner wave occurs. Understandably, wave measurements are often installed for some specific purpose other than understanding wave processes. Thus, there has not been sufficient useful wave data available anywhere to provide effective statistics for making inference on the frequency of occurrence of freaque waves. At the present, the assessment on the occurrence of freaque waves has basically advanced from regarding them as "rare" in the early days to some recent media suggestions that they are "far more frequent" than previously thought - all through pure speculations. Even though the reporting of freaque waves has become a media favorite, how often it occurs remains a simple question without an answer - and it is unlikely that can be answered any time soon. An answer to this question can only be gained from a long-term wave measurement program, which, at present, does not exist - except may be the newer work of Zakharov [9] asking how probability for freaque wave formation can be found through a theoretical study of "almost" 1-D water waves. 


\section{- Is there a life cycle for freaque wave occurrence?}

For the kind of freaque wave that "appears from nowhere and disappears without a trace" it clearly has a life cycle of only a few seconds. For the cases of a wall of water or a hole in the sea, their life cycle could be considerably prolonged. Either way, the question of life cycle for freaque waves has never been asked before, but it is nevertheless an interesting one - and may be used to distinguished different kind of freaque waves. It is also needed to stress the importance of spatial wave measurement. The current conventional wave measurements at a single point are clearly incapable of examining the life cycle of a freaque wave occurrence. But the life cycle question has not been a current concern, except maybe to those who work with optical freaque waves. Nonetheless, it is another interesting unexplored question.

\section{- Do freaque waves ever make loud noises?}

Sound or loud noise that comes with waves, particularly freaque waves, is another unexplored topic in ocean waves research. Liu et al. [4] described the experience of a young sailor who personally encountered a freaque wave on March 29, 2009 near the western tip of Australia during his solo sail around the world as a "never previously mentioned characterization of a freaque wave." ??? what about the noise??? That was wrong - a noisy freaque wave case has been previously mentioned (Liu [10]). We were being rightfully corrected by our friend and fellow ocean wave aficionado, Dr Luigi Cavaleri, of CNR-ISMAR, Italy, who had also a personal encounter with a freaque wave and recalled his experience in the book by Holthuijsen [11].

So it seems to be a well-documented and corroborated fact that noise does appear to have accompanied some freaque waves. However that's clearly not for all freaque waves. Dr Al Beeton, retired former Director of NOAA/GLERL, USA, had a vivid memory of an encounter with a "hole in the sea" kind of freaque wave in Lake Michigan many years ago, in 1956, when he was a young scientist. He does not explicitly recall any large noise connected to his experience. So, clearly, noise may have accompanied some freaque waves but not others. The sound effect in the freaque wave process is certainly worth looking into - hopefully, through concerted ocean wave measurements. Underwater sound has been well researched; maybe it's now time to assess their effects on ocean surface waves. At the very least, do we truly know freaque waves make a louder noise or sound effect than nonfreaque waves?

\section{- What is the role of wave breaking in connection with freaque wave occurrence?}

Zakharov [9] in his talk given at the Boris Chirikov Memorial Seminar had this to say in one of his slides: There are two types of rare catastrophic 
events on the ocean surface: 1. Freak waves (major catastrophic event); and 2. Wave breaking (minor catastrophic event). At the bottom of that slide there is this "Analytic theory for both of these are not developed." This is quite interesting to see some discussion between wave breaking and freaque waves. Even though Zakharov considered that breaking waves play a minor role from an analytical view, no one really knows how freaque waves and breaking waves are connected. Particularly if the existence of breaking waves will serve to enhance or decrease the intensity of a freaque wave another question that not even detailed measurements can expect to answer satisfactorily. In addition, Papadimitrakis and Dias [12] made statistical analysis on the general breaking of extreme waves in deep water with respect to various parameters. Their study pertains to the conventional deep water single point wave measurement as usual. All the academic theoretical analysis and conjectures are interesting. On the other hand, however, no one can really say if there was or was not breaking wave involvement during freaque waves. Therefore, conjecture abounds, and we still need actual measurements.

\section{- Are freaque waves predictable?}

In practice, all natural phenomena are predictable, if we know enough about them. If we don't have enough knowledge and are nevertheless still trying to make predictions, that is impressive and courageous, but no amount of analytical simulation can substitute for the real phenomenon. We understand there are operational freaque wave predictions already in place. But without practical measurement, how are the predictions being verified? For the time being we still do not know where, when, why, or how freaque waves happen. Mariners will be on their own if they ever encounter a freaque wave out there. Since we can only characterize the occurrence of freaque waves as rare or frequent, we cannot even provide a practical frequency of occurrence for them based on actual measurements, we must regard an answer to this simple question as negative. In our opinion, we just do not know enough at the present about freaque waves in the ocean. Ocean freaque waves are, in fact, not predictable.

\section{- How to realistically measure freaque waves?}

The answer to this question is also generally negative. But we wish to present a very positive prospect for a change. Something exciting in the wave measurement realm is happening in this second decade of the $21^{\text {st }}$ century.

A year ago, Bechle et al. [13] presented a paper at the annual Conference on Great Lakes Research in which they outlined a systematic procedure for possible measurement when a freaque wave is encountered. The approach makes use of the ATSIS system (Wanek and $\mathrm{Wu}$ [14]) for spatial wave measurement. When a freaque wave is encountered, it will go back to the 
spatial data and sort out the detailed data of the spatial freaque waves. So it cannot make the measurement in real time, but it is an inspiring first step that single point wave measurement will never be able to do.

The really difficult question is: will the freaque wave and ocean wave community ever pay attention to this "small step" for wave measurement after 70 years of complacency and contentment with measurements recorded from a single point in the vast ocean?

\section{What are the theoretical view points?}

We have so far been concerned mainly from observational viewpoints. The major advancements since freaque waves became a favorite topic of the general public and news media has been mostly in the theoretical arena. As Akhmediev and Ankiewicz [6] pointed out; in the title of their manuscript "Waves that appear from nowhere and disappear without a trace" can be applied to two objects: the rogue waves in the ocean and rational solutions of the nonlinear Schrodinger equation (NLSE). Indeed solving NLSE has been the backbone of the modern freaque wave study. The only real issue is the existence of the Draupner wave profile, nothing else seems to matter.

In 2010, the editors of the European Physical Journal conceived an issue of Special Topics (Ruban et al. [15]) by asking a selected special group of prominent physicists for their opinion on rogue waves. The editors posed these questions:

1. Is the phenomenon of "rogue waves" linear or nonlinear?

2. What is the onset of appearance of "rogue wave"? Is it the phenomenon related to modulation instability?

3. What is the spectral content of "rogue waves"?

4. How important is the distribution of wave amplitudes in registering rogue waves? For example, observations in optics pay special attention to the function of distribution.

5. Do you consider some other questions to be more important than those listed above?

This Special Topics issue has gathered opinions from 18 of the world's leading nonlinear physicists, and has provided very interesting and educational discussion and debates. They are clearly not really concerned about observations. These theoreticians in their pursuit brought out a whole body of knowledge of their own. But for a non-theoretical freaque wave aficionado, an immediate question that comes to mind is: where is the ocean?

Judging from the question about spectral contents, it appears that most of these theoreticians' concerns are generally connected to processes at a single point. Indeed it seems the whole field of freaque wave research in nonlinear physics has stemmed from the popularization of the Draupner wave data - a conventional wave measurement at a single-point location on the Statoil's 
Draupner Platform in the North Sea. So, while observations and measurements can bring different kinds of freaque waves, for theoreticians, the Draupner wave alone would seem to be sufficient.

\section{Discussion}

The editorial of the 2012 New Year issue of New Scientist Magazine has a very interesting byline: "Next year let's deal with world as it is, not as we would like it to be." It seems the whole science establishment, academic and others alike can benefit from this advice. When we are preoccupied in solving intriguing complicated formulas, the real world can sometimes become a minor inconvenience!

Furthermore, we have repeatedly alluded to the need of 3-D spatial wave measurements, what difference do they make?

Here are some thoughts between 1-D and 3-D spatial fields:

- Crests or troughs occur at 1-D are not necessarily the crests or troughs in the 4-D spatial field.

- A maximum wave height in 1-D is not necessarily the maximum wave height in 4-D spatial wave field.

- If there is no freaque wave found in the 1-D data it does not mean there is no freaque wave in the 4-D spatial wave field.

- As there can be clear defined zero level in 1-D so that we can talk about zero-crossing cases. There is no equivalence in the 4-D spatial field.

- It is possible to readily sensing wave breaking effects in 4-D, but not in the 1-D wave field.

So, in reality, we cannot expect true processes of ocean waves to emerge from exploring waves and freaque waves with only the 1-D wave field and with waves only measured from a single point location.

\section{Concluding remarks}

A well-known quote that was attributed to the 18th century mathematician, Pierre-Simon Laplace (1749-1827), is: "What we know is not much. What we do not know is immense." (Ce que nous connaissons est peu de chose; ce que nous ignorons est immense.) It seems this quote is very applicable to confront the challenges on our study on freaque waves. Now that we have managed to examine both what we DO know and what we DO NOT know on freaque waves, we must admit that we have immense admiration for Laplace' sagacious observations. What was also interesting is that in both cases, we have invariably arrived at the same conclusion- we need more intensive and modernized spatial ocean wave measurement! The destitution of relevant wave measurement for freaque waves study is certainly nothing new or trivial. Nonlinear physics studies 
have greatly contributed to the development, advancement, and popularization of our modern freaque wave studies. But the conventional wave measurement system is still relying on the last conceptual advancement, vintage 1945. A new conceptualization is certainly long overdue. Yes, we are over a decade into $21 \mathrm{st}$ century; the Draupner wave form that was discovered at the end of last century cannot sustain our nonlinear physics research indefinitely. There is so much we still do not know and should not be pretending that they don't exist. We should be at least contemplating about something like spatial wave measurement by now to confront the unrelenting challenges we face!

\section{Acknowledgement}

This is GLERL Contribution No. 1653.

\section{References}

[1] Draper, L., 1964, "Freak" Ocean Waves, Oceanus X:4.

[2] Haver, H., 2004, "A possible freak wave event measured at the Draupner Jacket January 1 1995”, Proceedings, Rogue Waves 2004, Ifremer.

[3] Dias, F., T.J. Bridges, and J.M. Dudley, 2010, "Rogue Waves", Lecture Note Series, IMS, NUS - Review Volume.

[4] Liu, P.C., C.H. Wu, A.J. Bechle, K.R. MacHutchon, and H.S. Chen, 2010, Brief communication "What do we know about freaque waves in the ocean and lakes and how do we know it? Nat. Hazards Earth Syst. Sci., 10, 21912196.

[5] Liu, P.C., C.H. Wu, A.J. Bechle, H.S. Chen, and K.R. MacHutchon, 2012, "What do we not know about freaque waves in the ocean and lakes and where to go from here?", Proceedings of the ASME 2012 31st International Conference on Ocean, Offshore and Arctic Engineering OMAE2012.

[6] Akhmediev, N., A. Ankiewicz, M. Taki, 2009, "Waves that appear from nowhere and disappear without a trace", Physic Letters A, 373, 675-678.

[7] Hohmann, R., U. Kuhl, H.J. Stockmann, L. Kaplan. and E.J. Heller, 2010, "Freak waves in the linear regime: A microwave study", Phys. Rev. Lett, 104, 093901.

[8] Chabchoub, A., N.P. Hoffmann, and N. Akhmediev, 2012, “Observation of rogue wave holes in a water wave tank", J. Geophys. R. 117, C00J02, doi:10.1029/2011JC007636.

[9] Zakharov, N.E., 2008, "Freak Waves and Wave Breaking - Catastrophic Events in Ocean", Talks at Chirikov Memorial Seminar at BINP - 23 May 2008 (http://www.quantware.upstlse.fr/chirikov/memorialsem.html).

[10] Liu, P.C., 2011, Corrigendum to brief communication "What do we know about freaque waves in the ocean and lakes and how do we know it?", Nat. Hazards Earth Syst. Sci., 11, 331-332.

[11] Holthuijsen, L.H., 2007, "Waves in Oceanic and Coastal Waters", Cambridge University Press, 404 pp., 2007. 
[12] Papadimitrakis, I.A. and F. Dias, 2008, "Occurrence and breaking of extreme waves in deep water, A stochastic approach revisit", Proceedings, Rogue Waves 2008. Ifremer.

[13] Bechle, A.J., P.C. Liu, and C.H. Wu, 2011, "Monitoring and characterization of freak waves in Lake Superior." Presented at the 54th annual Conference on Great Lakes Research, Duluth, Minnesota.

[14] Wanek, J.M. and C.H. Wu, 2006, "Automated trinocular stereo imaging system for the three dimensional surface wave measurements." Ocean Engineering, 33, 723-747.

[15] Ruban, V., Y. Kodama, M. Ruderman, J. Dudley, R. Gramshaw, P.V.E. McClintock, M. Onorato, C. Kharif, E. Pelinovsky, T. Soomere, G. Lindergren, N. Akhmediev, A. Slunyaev, D. Solli, C. Ropers, B. Jalali, F. Dias and A. Osborne, 2010, "Rogue waves - towards a unifying concept?: Discussions and debates, Eur. Phys. J. Special Topics, 185, 5-15. 